\title{
OPTICALLY DETECTED ZERO FIELD MAGNETIC RESONANCE OF PHOSPHOROUS ACTIVATED ALKALI HALIDE CRYSTALS
}

\author{
S.J. HUNTER, K.W. HIPPS and A.H. FRANCIS \\ Department of Chemistry, The University of Michigan, \\ Ann Arbor, Michigan 48109, USA
}

Received 14 June 1977

\begin{abstract}
Optically detected zero field magnetic resonance (ODMR) has been utilized to obtain the fine structure parameters $(D i$ and $|E|$ of the phosphorescent color center in phosphorous activated potassium chloride and bromide single crystals. The dipole activity of the zero field spin-orbital states, the Franck-Condon analysis of the vibronic structure of the phosphorescence spectrum and the fine structure parameters are all consistent with the proposed identification of the impurity center as $\mathrm{PO}_{2}^{-}$ion.
\end{abstract}

\section{Introduction}

Single crystals of alkali halides grown from the melt in the presence of phosphorous vapors exhibit both a narrow line and broad band luminescence that has variously been attributed to inclusion of elemental phosphorous, $\mathrm{PO}_{2}$ radical and $\mathrm{PO}_{2}^{-}$molecular ion $[1-4]$. Recently, several detailed investigations of the electronic and vibrational spectra of the phosphorous activated alkali halide lattices have been reported which argue strongly in favor of the identification of the impurity center responsible for the narrow line emission as the $\mathrm{PO}_{2}^{-}$ion $[5,6]$. The correct identification of the species responsible for the observed narrow line emission is of particular interest since there are relatively few examples of molecular color centers in alkali halide lattices which exhibit a line spectrum. The identification of the impurity center as $\mathrm{PO}_{2}^{-}$rests heavily upon the close similarity between the observed electronic spectrum and the spectra of the isoelectronic species $\mathrm{NO}_{2}^{-}[7-$ 9] and $\mathrm{SO}_{2}[10,11]$.

At high concentrations of phosphorous, lattices activated by both elemental phosphorous and $\mathrm{PO}_{2}^{-}$ion are evidently obtained [5]. Elemental phosphorous is thought to be responsible for the rather broad and featureless emission which partially overlaps the region of narrow line emission.
It is the purpose of the present work to examine the narrow line emission spectrum of phosphorous activated potassium chloride and bromide single crystals using the technique of optically detected magnetic resonance (ODMR) $[12,13]$ in an attempt to more completely characterize the nature of the impurity center.

\section{Experimental}

Single crystal samples of phosphorous activated $\mathrm{KCl}$ and $\mathrm{KBr}$ were prepared by the method described by Plachenov et al. [4] . High purity (99.99\%) alkali halide together with $0.02 \%$ by weight of red phosphorous was sealed under vacuum in a vitreous silica tube. The sample tube was then lowered at a rate of approximately $1 \mathrm{~cm} / \mathrm{h}$ through a high temperature Bridgeman furnace. In this manner large single crystals of the phosphorous activated alkali halide were obtained. Crystals were cleaved parallel to the principal cleavage directions of the cubic lattices, mounted in a slow wave microwave helix attached to the end of a length of semirigid cryogenic coaxial cable and cooled to the working temperature by either a $1 \mathrm{~atm}$ helium exchange gas or direct immersion in superfluid liquid helium.

The source of frequency swept microwave radiation was a Hewlett-Packard 8600B sweeper and associated 
plug-in modules. Lifetime measurements were made using the doubled $5900 \AA$ output from a pulsed Chromatix $\mathrm{CMX}-4$ dye laser. Decay curves for the lifetime measurements were averaged with a Northern NS575 CAT. Continuous excitation for the ODMR and optical spectra was provided by the ultraviolet output of a $1 \mathrm{~kW}$ mercury-xenon high pressure arc lamp. Phosphorescence was dispersed with a l-meter Jarrell-Ash spectrometer and detected with a refrigerated EMI 9558-QB photomultiplier.

\section{Results and discussion}

In the following discussion we shall adopt the molecular axis system with $x$ normal to the molecular plane and $z$ parallel to the two-fold axis. The $\mathrm{PO}_{2}^{-}$ion has $\mathrm{C}_{2 \mathrm{r}}$ symmetry and is isoelectronic with the nitrite ion and sulfur dioxide, both of which have been extensively studied spectroscopically. According to simple molecular orbital theory and by analogy to $\mathrm{NO}_{2}$ and $\mathrm{SO}_{2}$, the lowest triplet state of the $\mathrm{PO}_{2}^{-}$ion would be of orbital symmetry $B_{1}$, arising from promotion of an electron from a non-bonding s-type orbital localized on phosphorous, to a $\pi^{*}$ orbital which is anti-bonding with respect to the $\mathrm{P}-\mathrm{O}$ bond. As the bond angle is increased from $90^{\circ}$ to $180^{\circ}$ the initial phosphorous orbital tends from pure s-type to p-type and the transition energy decreases.

The individual spin-orbital symmetries are $A_{1}\left(\tau_{y}\right)$, $\mathrm{A}_{2}\left(\tau_{x}\right)$ and $\mathrm{B}_{2}\left(\tau_{z}\right)$. Transitions from the $\tau_{y}$ and $\tau_{z}$ spin-orbital states are electric dipole allowed by first order spin-orbit coupling selection rules with $z$ and $y$ polarizations, respectively. Transitions from the $\tau_{x}$ spin-orbital state are electric dipole forbidden in first order spin-orbit coupling in the absence of a nontotally symmetric vibration. Again, by analogy to both $\mathrm{NO}_{2}^{-}[8,9]$ and $\mathrm{SO}_{2}[10,11]$ we anticipate that the most active spin-orbital state will be $\tau_{z}$ which exhibits a large direct spin-orbit coupling matrix element (onecenter) with the strongly allowed ${ }^{1} B_{2}$ state of $\pi \pi^{*}$ type.

In nitrite ion the $\tau_{y}$ spin-orbital state is only very weakly active. As judged from the relative intensities of the appropriate high field Zeeman lines with the field parallel to the $y$ molecular axis, this activity is approximately $10 \%$ that of the $\tau_{z}$ spin-orbital level.

Since the spectroscopic and ODMR results obtained
Table 1

\begin{tabular}{llll} 
& $\mathrm{NO}_{2}^{-}$ & $\mathrm{SO}_{2}$ & $\mathrm{PO}_{2}^{-}$ \\
\hline$|D| \mathrm{cm}^{-1}$ & $0.63 \mathrm{a})$ & $\left.0.275^{\mathrm{b}}\right)$ & $0.202(0.1953) \mathrm{c})$ \\
$|E| \mathrm{cm}^{-1}$ & 0.23 & 0.0158 & $0.0488(0.0501)$ \\
$\tau_{z}(\mathrm{~ms})$ & 3.1 & 2.4 & 6 \\
$\tau_{y}$ & & 12 & 90 \\
$\tau_{x}$ & & 41 & \\
$k_{1}^{2}$ & 0.85 & 0.79 & 0.92 \\
$k_{2}^{2}$ & 0.71 & 0.70 & 0.97 \\
$b_{1}$ & & 1.63 & 1.05 \\
$b_{2}$ & & 1.60 & 1.67 \\
\hline
\end{tabular}

a) Data taken from refs. $[8,9]$.

b) Data taken from ref. [11] $\tau_{x}$ and $\tau_{y}$ lifetimes are influenced by spin-lattice relaxation.

c) Values in parentheses refer to bromide lattice.

from $\mathrm{KCl}$ and $\mathrm{KBr}$ lattices were essentially equivalent, the following discussion, for convenience, refers only to the halide lattice. The $|D|$ and $|E|$ values obtained from both lattices are given in table 1 .

Monitoring the most intense zero-phonon band at approximately 4090 \& (see fig. 2), two moderately strong ODMR signals were observed at $2930 \mathrm{MHz}$ and $4580 \mathrm{MHz}$, both corresponding to an increase in phosphorescence intensity. The resonance linewidths were approximately $9 \mathrm{MHz}$. Spin-alignment evidently persists well above $4 \mathrm{~K}$ and ODMR signals could easily be obtained at this temperature. Measurements by Avarmaa [5] of the temperature dependence of the phosphorescence lifetime of this impurity center suggest that spin alignment is obtained at approximately $12 \mathrm{~K}$. Using microwave power levels in excess of $2 \mathrm{~W}$, it was not possible to observe any indication of chlorine quadrupole splitting indicating that the impurity center does not involve significant covalent bonding with the halogens. A third resonance at $7510 \mathrm{MHz}$ could be observed by simultaneousiy pumping (EEDOR) the $2930 \mathrm{MHz}$ resonance. The ODMR results are consistent with either of the relative level ordering schemes depicted in fig. 1 .

The lifetimes of the individual spin states were obtained by the method of microwave induced delayed phosphorescence $[14,15]$ and were found to be $T_{y} \approx$ $T_{x}=2 \mathrm{~ms}$ and $T_{z}=90 \mathrm{~ms}$. These results are in approximate agreement with those obtained by Avarmaa [5] from graphical decomposition of the low temperature total phosphorescence decay curve and confirm his interpretation of the multiple lifetime decay as arising from individual spin-states of the spin-aligned triplet. 


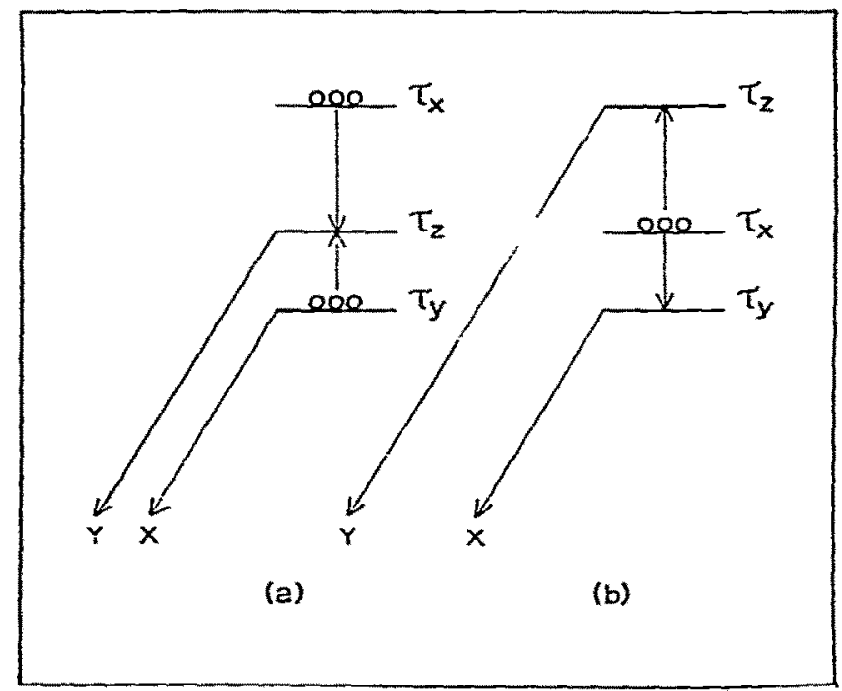

Fig. 1. Schematic level ordering schemes for the proposed $\mathrm{PO}_{2}^{-}$ color center, indicating approximate relative steady state spin distributions.

In the microwave induced delayed phosphoresceñce experiment, it was observed that microwave pumping of the $4580 \mathrm{MHz}$ transition did not attenuate the longlived phosphorescence component, whereas microwave pumping of the $2930 \mathrm{MHz}$ transition markedly attenuated the long-lived component. This observation suggests that one of the spin-orbital levels is totally inactive and does not contribute to the observed phosphorescence intensity. This observation is consistent with the radiative decay scheme illustrated in fig. 1 a but inconsistent with that of fig. $1 \mathrm{~b}$, and accordingly, we adopt the former scheme in subsequent discussion.

We have obtained absolute values of the zero-field splitting parameters $D$ and $E$ from the energy level scheme in fig. 1a. These are compared with the zerofield parameters of $\mathrm{NO}_{2}^{-}$[9] and $\mathrm{SO}_{2}$ [10] in table 1 . It will be observed that the suggested relative level ordering scheme is identical to that established for $\mathrm{NO}_{2}^{-}$by high field Zeeman measurements [9]. The zero-field splitting parameters of $\mathrm{PO}_{2}^{-}$and $\mathrm{SO}_{2}$ are quite comparable in magnitude and considerably smaller than those of $\mathrm{NO}_{2}^{-}$. Relatively large one-center spinspin dipolar interaction matrix elements on the apex phosphorous atom are expected to account for the major portion of the zero-field splitting.

The phosphorescence and $\mathrm{PMDR}$ spectra of $\mathrm{PO}_{2}^{-}$ centers in $\mathrm{KCl}$ host lattice at $2 \mathrm{~K}$ are illustrated in fig. 2. The vibrational frequencies of the $\mathrm{PO}_{2}^{-}$ion have

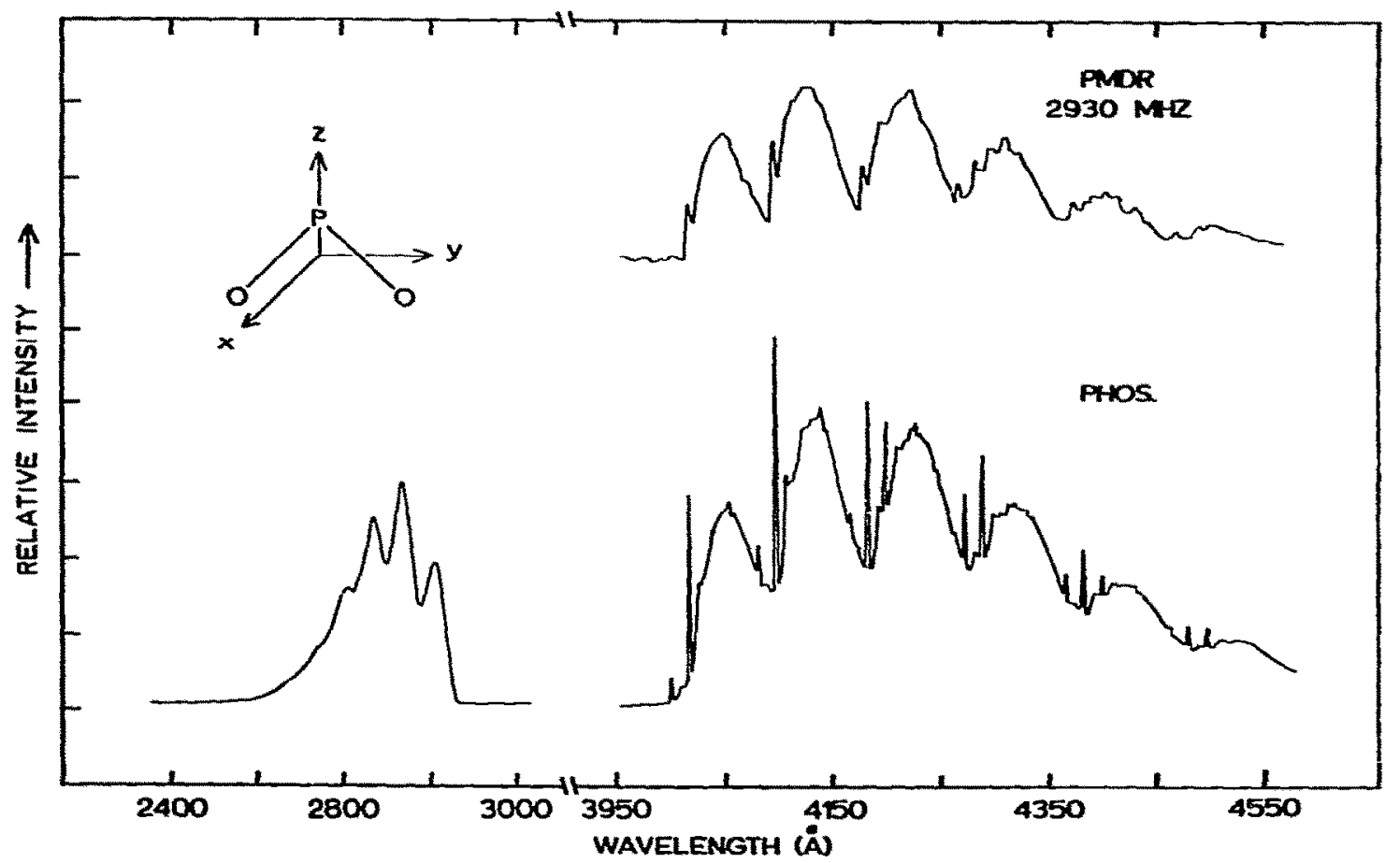

Fig. 2. The phosphorescence excitation, phosphorescence and phosphorescence-microwave double resonance (PMDR) spectra of a phosphorous activated $\mathrm{KCl}$ single crystal. 
been determined by infrared spectroscopy in a number of host alkali halide lattices. The values obtained for $\mathrm{KCl}$ host lattice are typical [16]: $\nu_{1}=1094 \mathrm{~cm}^{-1}$ (sym.st.), $\nu_{2}=501 \mathrm{~cm}^{-1}$ (sym. bend.) and $\nu_{3}=1207 \mathrm{~cm}^{-1}$ (asym. st.). The phosphorescence spectrum consists of vibrational progressions in both $\nu_{1}$ and $\nu_{2}$ with the intensity maximum in the second and first members of each progression, respectively, indicative of both an increase in the OPO bond angle and an increase in the $\mathrm{PO}$ bond length in the excited state. This is consistent with both the predictions of Mulliken [17] and Walsh [18] and with the observed decrease of the frequency of $\nu_{1}$ in the ${ }^{1} B_{2}$ state. A Franck-Condon analysis of the vibrational structure was performed using the corrected peak intensities of the individual mode progressions. Since at $4.2 \mathrm{~K}$ emission occurs from the lowest vibrational level of the excited state:

$\frac{I\left(0, \nu_{n}^{i}\right) \nu_{0 n}^{4}}{I(0,0) / \nu_{00}^{4}}=\frac{\left.!\left\langle u_{0}^{i} \mid u_{n}^{i}\right\rangle\right|^{2}}{\left|\left\langle u_{0}^{i} \mid u_{0}^{i}\right\rangle\right|^{2}}$

where $i$ represents the normal mode under consideration, $n$ designates the vibrational level of the ground state, and $u_{n}$ is the vibrational wavefunction of the $n$th leviel. Eq. (1) assumes the normal coordinates for ground and excited states are parallel. Equations for the individual vibrational overlap integrals $\left|\left\langle u_{0} \mid u_{n}\right\rangle\right|^{2}$ can be generated using the general formula derived by Hipps et al. [19].

The overlap integrals of eq. (1) are dependent on four parameters, $k_{i}^{2}$ and $b_{i}$, where $i$ again labels the mode in question, $k_{i}^{2}$ is the ratio of the excited to the ground state frequencies of the $i$ th mode and $b_{i}$ is the normalized displacement of that mode. In the case of the stretching mode, only three lines of adequate intensity were available and, although this is sufficient for an algebraic determination of the parameters, no check on thcir accuracy was possible. The bending mode, however, furnished five usable lines and these were fit to $4 \%$ precision by variation of $k_{i}^{2}$ and $b_{i}$. The results of this procedure are presented in table 1 and are consistent with our expectation that the apex angle and $\mathrm{P}-\mathrm{O}$ bond length both increase in the excited state.

\section{Conclusion}

The microscopic details of color centers in alkali halide crystals are often quite complex and, accordingly, we feel compelled to carefully summarize the conclusions of this work. We have confirmed the lifetime measurements and spectroscopic measurements made by Avarmaa [5]. Additionally, the ODMR results are consistent with his suggestion that the multiple lifetime decay observed below about $12 \mathrm{~K}$ is due to an increasing spin alignment of a zero-field triplet. We have obtained the absolute magnitudes of the triplet zerofield splitting parameters $|D|$ and $|E|$.

All of the above data is consistent with the identification of the narrow line luminescent impurity center in phosphorous activated alkali halide lattices as $\mathrm{PO}_{2}^{-}$. Other details of the microscopic model remain uncertain: for example, the position and orientation of the impurity. We are initiating an investigation of the magnetic field angular dependence of the ODMR spectrum in order to ascertain the orientation of the $\mathrm{PO}_{2}^{-}$impurity center.

\section{References}

[1] G.A. Mikhal'chenko, Izv. Akad. Nauk SSSR Ser. Fiz. 29 (1965) 40.

[2] V.P. Avdonin, I.A. Vasil'ev, G.A. Mikhal'chenko, B.T. Plachenov and V.A. Shibaex, Fiz. Tverd. Tela. 7 (1965) 3110.

[3] B.T. Plachenov, V.P. Aydonin, G.A. Mikhal'chenko and V.I. Smagin, Izv. Akad. Nauk SSSR. Ser. Fiz. 30 (1966) 1411.

[4] B.T. Plachenov, V.P. Avdonin, V. Kapishovskii and G.A. Mikhal'chenko, Izv. Akad. Nauk SSSR Ser. Fiz. 31 (1967) 2025.

[5] R.A. Avarmaa, Optika i Spektroskopiya 32 (1972) 959.

[6] R.A. Avarmaa, Izv. Akad. Nauk SSSR 36 (1972) 1066.

[7] R.A. Avarmaa and K. Rebane, Izv. Akad. Nauk SSSR, to be published; Phys. Stat. Sol. 35 (1969) 107;

T. Timusk and W. Staude, Phys. Rev. Letters 13 (1964) 373.

[8] R.M. Hochstrasser and A.P. Marchetti, J. Chem. Phys. 50 (1969) 1727.

[9] R.M. Hochstrasser and A.H. Zewail, J. Chem. Phys. 54 (1971) 2979.

[10] R.M. Hochstrasser and A.P. Marchetti, J. Mol. Spectry. 35 (1970) 335.

[11] D.S. Tinti, Chem. Phys. Letters 12 (1971) 169.

[12] M. Sharnoff, J. Chem. Phys. 46 (1967) 3263. 
[13] J. Schmidt and J.H. van der Waals, Chem. Phys. Letters 2 (1968) 640.

[14] J. Schmidt, W.S. Veeman and J.H. van der Waals, Chem. Phys. Letters 4 (1969) 341.

[15] D.A. Antheunis, J. Schmidt and J.H. van der Waals, Chem. Phys. Letters 6 (1970) 255.
[16] K.K. Rebane and L.A. Rebane, Pure Appl. Chem. 37 (1974) 161 .

[17] R.S. Mulliken, Rev. Mod. Phys. 14 (1942) 204.

[18] A.D. Walsh, I. Chem. Soc. (1953) 226.

[19] K.W. Hipps, G.A. Merrell and G.A. Crosby, J. Phys. Chem. 80 (1976) 2232. 